\title{
Galaxy superclusters and cosmic strings
}

\section{from Craig Hogan}

OBSERVATIONAL studies of superclusters - the largest aggregations of galaxies in the Universe - have become increasingly quantitative in recent years, putting a considerable strain on many theoretical models that are unable to account for strong clustering on very large scales. It is thus a considerable achievement for the string theory of galaxy formation that it has now been shown to explain some of the most puzzling observations of superclustering in a remarkably parameter-free way (Turok, N. Phys. Rev. Lett. 55, 1801; 1985).

When the Palomar sky survey was completed in the 1950s, G. Abell examined it by eye and catalogued thousands of the densest aggregations of galaxies, now called Abell clusters. It has since been confirmed that these clusters are themselves clustered on larger scales. The observational controversy about the statistical properties of Abell cluster correlations continues, but little doubt remains about the essential facts: Abell clusters are more strongly clustered than galaxies themselves by a factor of at least ten; and their correlations extend out to much larger scales, again, perhaps a factor of ten in length.

If these correlations are intrinsic (and not the effect of, say, a subtle observational selection effect in Abell's catalogue), the implications are striking. The clusters and the galaxies cannot both be regarded as tracers of a single underlying mass distribution, or their correlations would be the same. Therefore, some factor other than pure gravitational clustering of galaxies must be added to the theory of clustering. It may be possible to explain the correlations in terms of the different types of local conditions and processes that contribute to the creation of a cluster or a galaxy. Thus, galaxies and clusters may represent differently 'biased' samples of the same underlying mass distribution (Kaiser, N. Astrophys. J. 284, L9; 1984). Or, as Turok now proposes, it may be that the phenomenon is a manifestation of something radically different.

As I have previously discussed in these columns (Nature News and Views 310, 365 ; 1984) cosmic strings are a natural cosmological by-product of certain unified gauge theories corresponding roughly to magnetic flux tubes or superfluid vortices in the cosmic vacuum. Their multifarious unusual gravitational side-effects may include the formation of galaxies (for a review, see Vilenkin, A. Phys. Rep. 121,
$263 ; 1985)$. Cosmic strings are formed as a dense net of pseudo-random walks, but quickly evolve as the Universe expands into a thin net of infinite (open) strings and a debris of closed oscillating string loops that have broken off from the net. It is the loops which are thought to form galaxies by gravitationally accumulating matter around themselves. Because the

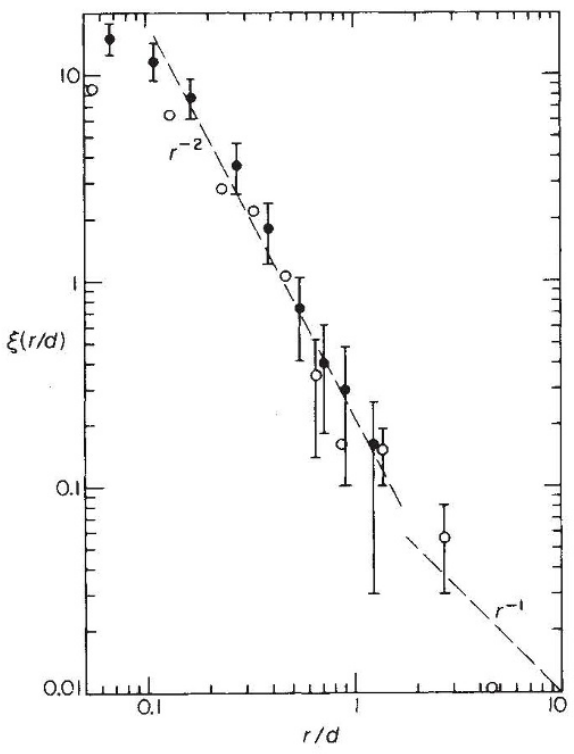

Two-point spatial correlation function $\zeta$ for Abell clusters (open circles) and for cosmic string loops (filled circles) as a function of $r / d$, where $r$ is the separation and $d$ is the mean separation of the objects considered. Error bars in the data for Abel clusters correspond to the square root of the number of pairs in each separation bin (assuming Poisson statistics). Error bars in string simulations correspond to the standard deviations of the mean for 18 independent simulations (from Phys. Rev. Lett. 55, 1802; 1985).

locations of the loops are not determined by gravity, but rather by where and how they broke off from the net of open strings, unusual correlations are expected to be found in the locations of galaxies and clusters. It is these correlations which Turok has now calculated.

Usually correlations among galaxies are thought to be induced by the fact that they attract one another. In the string picture, gravity may play a part in galaxy correlations, but cluster correlations are unlikely to be caused by cluster-cluster attraction but rather by the fact that the string loops causing cluster formation often came from the splitting up of a larger parent loop, and are therefore correlated because of the (non-gravitational) tension forces within the parent. Such string correlations can be introduced over very large scales because cosmic-string tension is so large that disturbances travel along strings at the speed of light.

To the extent that the statistical properties of the string system evolve selfsimilarly in time, a scale-free autocorrelation is expected to develop among string loops. Suppose loops of a given size are examined and their correlation length (roughly, the typical size of clusters of the loops) calculated. Then, in a scale-free system, one should find that the correlation length of larger or smaller loops measured at an appropriately scaled epoch should scale as their size, as should their mean separation. This behaviour is in qualitative agreement with what is found - larger, rarer systems such as clusters have larger correlation lengths than smaller, commoner systems such as galaxies. This argument does not establish the ratio of lengths for any given scale or the form of the correlation function, but these details can be calculated by computer. The relativistic equations of motion for strings must be integrated, including the exchange of partners for intercommuting of strings when they cross. By doing this for many initial configurations, it is possible to verify that the network indeed tends towards a state evolving with statistical self-similarity in time, and it is possible to measure the correlations in this state.

A sophisticated computer program has been developed by A. Albrecht and Turok (Phys. Rev. Lett. 54, 1868; 1985) to simulate in this way the evolution of the string network and the formation of loops. Turok then examined the distribution of large loops (large enough to be seeds for the formation of Abell clusters) and calculated their correlation function. Remarkably, his result agrees both in functional form and amplitude with oservational results for the Abell cluster autocorrelation (see figure).

It is almost incredible that such a simple prescription (even if the calculational implementation is anything but simple) immediately produces the desired result. It demonstrates the predictive power of the string theory. As calculations become more refined, the theory is likely to confront observation more forcefully. We can expect to see more detailed predictions, such as the correlations of various Abell richness classes, incorporating detailed models of processes such as the gravitational accretion of clusters around loops and the effects of gravitational radiation recoil on loop accretion and correlations. Turok's interesting result provides a framework for such studies.

Craig Hogan is at the Steward Observatory, University of Arizona, Tucson, Arizona 85721 , $U S A$. 